MATHEMATICS OF COMPUTATION

Volume 68, Number 228, Pages 1447-1463

S 0025-5718(99)01094-7

Article electronically published on May 19, 1999

\title{
APPROXIMATION OF THE VIBRATION MODES OF A PLATE BY REISSNER-MINDLIN EQUATIONS
}

\author{
R. G. DURÁN, L. HERVELLA-NIETO, E. LIBERMAN, \\ R. RODRÍGUEZ, J. SOLOMIN
}

\begin{abstract}
This paper deals with the approximation of the vibration modes of a plate modelled by the Reissner-Mindlin equations. It is well known that, in order to avoid locking, some kind of reduced integration or mixed interpolation has to be used when solving these equations by finite element methods. In particular, one of the most widely used procedures is the mixed interpolation tensorial components, based on the family of elements called MITC. We use the lowest order method of this family.

Applying a general approximation theory for spectral problems, we obtain optimal order error estimates for the eigenvectors and the eigenvalues. Under mild assumptions, these estimates are valid with constants independent of the plate thickness. The optimal double order for the eigenvalues is derived from a corresponding $L^{2}$-estimate for a load problem which is proven here. This optimal order $L^{2}$-estimate is of interest in itself.

Finally, we present several numerical examples showing the very good behavior of the numerical procedure even in some cases not covered by our theory.
\end{abstract}

\section{INTRODUCTION}

Finite element discretization of Reissner-Mindlin equations is, to date, the usual way to approximate the bending of an elastic plate of moderate or small thickness (see for instance $[4,13,19]$ ).

For load problems, because of the ellipticity of these equations, the classical theory ensures the convergence of standard finite element approximations. However these elements lead to wrong results when the thickness is small with respect to the other dimensions of the plate. This is because of the so-called locking phenomenon, which is now well understood (see for instance [7]). In order to avoid this drawback, reduced integration or mixed methods are usually applied. To perform their mathematical analysis, a family of problems, one for each thickness $t>0$, is considered and approximation results valid uniformly on $t$ are sought (see for instance $[2,7,10])$.

Received by the editor November 13, 1997.

1991 Mathematics Subject Classification. Primary 65N25, 65N30.

Key words and phrases. Mixed methods, Reissner-Mindlin, plates, eigenvalues.

The first author was partially supported by UBA through grant EX-071. Member of CONICET (Argentina).

The fourth author was partially supported by FONDECYT (Chile) through grant No. 1.960.615 and FONDAP-CONICYT (Chile) through Program A on Numerical Analysis.

The fifth author was partially supported by SECYT through grant PIP-292. Member of CONICET (Argentina). 
Among these methods, the so-called MITC ones, introduced by Bathe and Dvorkin in [6], are very likely the most used in practice. Their application to load problems has also drawn much attention from the mathematical point of view $([5,8,10,15,16])$. The aim of this paper is to analyze one of these methods, that of lowest order, when used to approximate the free vibration bending modes of a plate. Being non-conforming, the spectral theory based on minimum-maximum principles (see Section 8 of [3]) cannot be directly applied to this method. Instead, our analysis will be based on the abstract spectral theory for compact operators as stated in Section 7 of [3].

Optimal order of convergence in $H^{1}$ norm is known for the application of the lowest order MITC method to load problems $([8,10,16])$. This, combined with known regularity results $([2,7])$, allow us to prove analogous estimates for the approximation of the eigenfunctions in vibration problems. Let us remark that this would not be the case for higher order methods, since the eigenfunctions are in general not regular enough for us to attain similar results.

For conforming methods (which is not our case) the convergence of the eigenfunctions in $H^{1}$ directly yields a double order of convergence for the eigenvalues (Lemma 9.1 in [3]). Alternatively, when such double order holds for the $L^{2}$ convergence in the corresponding load problem without further assumptions on the regularity of the solution, abstract spectral theory can be used to prove a similar result even for non-conforming methods (see for instance Section 7 of [3]).

We prove such optimal (in order and regularity) $L^{2}$ error estimates for the lowest order MITC method, valid uniformly on the thickness $t$. This kind of estimates have been proved before for higher order MITC methods in $[8,15]$, but the arguments therein do not apply to our case. Thus, our results complete the analysis of the MITC elements.

In Section 2 we present the mathematical setting and state the spectral approximation results. The analysis carried out yields $t$-independent optimal error estimates for the approximation of eigenfunctions and eigenvalues. The proofs are valid for eigenvalues remaining uniformly separated from the rest of the spectrum as the thickness becomes small. These results rely on properties of the associated load problems, which are proved in Section 3. Finally, in Section 4, we present numerical experiments confirming the theoretical results and showing the good performance of the method. We also exhibit the potential applicability of this method to problems not covered by the theoretical analysis.

\section{Approximation of the eigenvalue problem}

Consider an elastic plate of thickness $t$ with reference configuration $\Omega \times\left(-\frac{t}{2}, \frac{t}{2}\right)$, where $\Omega \in \mathbb{R}^{2}$ is a convex polygonal domain. The deformation of the plate is described by means of the Reissner-Mindlin model in terms of the rotations $\beta_{t}=$ $\left(\beta_{t}^{1}, \beta_{t}^{2}\right)$ of its midplane $\Omega$ and the transverse displacement $w_{t}$ (see for example [7]).

Assuming that the plate is clamped, its free vibration modes are solutions of the following problem:

Find a non-trivial $\left(\beta_{t}, w_{t}\right) \in H_{0}^{1}(\Omega)^{2} \times H_{0}^{1}(\Omega)$ and $\omega_{t}>0$ such that

$$
\begin{array}{r}
t^{3} a\left(\beta_{t}, \eta\right)+\kappa t \int_{\Omega}\left(\nabla w_{t}-\beta_{t}\right) \cdot(\nabla v-\eta)=\omega_{t}^{2}\left[t \int_{\Omega} \rho w_{t} v+\frac{t^{3}}{12} \int_{\Omega} \rho \beta_{t} \cdot \eta\right], \\
\forall(\eta, v) \in H_{0}^{1}(\Omega)^{2} \times H_{0}^{1}(\Omega),
\end{array}
$$


where $\omega_{t}$ is the angular vibration frequency, $a$ is the $H_{0}^{1}(\Omega)^{2}$-elliptic bilinear form defined by

$$
a(\beta, \eta):=\frac{E}{12\left(1-\nu^{2}\right)} \int_{\Omega}\left[\sum_{i, j=1}^{2}(1-\nu) \varepsilon_{i j}(\beta) \varepsilon_{i j}(\eta)+\nu \operatorname{div} \beta \operatorname{div} \eta\right],
$$

$\varepsilon_{i j}(\beta)$ being the linear strain tensor, $E$ the Young modulus and $\nu$ the Poisson ratio, $\kappa:=E k / 2(1+\nu)$ is the shear modulus, with $k$ being a correction factor usually taken as $5 / 6$, and $\rho$ is the density.

The lowest vibration frequencies $\omega_{t}$ correspond to bending modes of the plate, and for each of them $\lambda_{t}:=\rho \omega_{t}^{2} / t^{2}$ attains a finite limit as $t$ goes to zero, as is shown below. Therefore it is convenient for the mathematical analysis to consider the following rescaled eigenvalue problem:

$$
\begin{aligned}
a\left(\beta_{t}, \eta\right)+\frac{\kappa}{t^{2}}\left(\nabla w_{t}-\beta_{t}, \nabla v-\eta\right) & =\lambda_{t}\left[\left(w_{t}, v\right)+\frac{t^{2}}{12}\left(\beta_{t}, \eta\right)\right], \\
\forall(\eta, v) & \in H_{0}^{1}(\Omega)^{2} \times H_{0}^{1}(\Omega),
\end{aligned}
$$

where $(\cdot, \cdot)$ denotes the standard $L^{2}$ inner product. Note that all the eigenvalues in (2.2) are strictly positive, because of the symmetry of the bilinear forms and the ellipticity of its left hand side (see [7]). as

Introducing the shear strain $\gamma_{t}:=\frac{\kappa}{t^{2}}\left(\nabla w_{t}-\beta_{t}\right)$, problem $(2.2)$ can be written

$$
\left\{\begin{array}{l}
a\left(\beta_{t}, \eta\right)+\left(\gamma_{t}, \nabla v-\eta\right)=\lambda_{t}\left[\left(w_{t}, v\right)+\frac{t^{2}}{12}\left(\beta_{t}, \eta\right)\right], \quad \forall(\eta, v) \in H_{0}^{1}(\Omega)^{2} \times H_{0}^{1}(\Omega), \\
\gamma_{t}=\frac{\kappa}{t^{2}}\left(\nabla w_{t}-\beta_{t}\right) .
\end{array}\right.
$$

In order to analyze the approximation of this eigenvalue problem it is convenient to introduce the operator

$$
T_{t}: L^{2}(\Omega)^{2} \times L^{2}(\Omega) \rightarrow L^{2}(\Omega)^{2} \times L^{2}(\Omega),
$$

defined by $T_{t}(\theta, f):=\left(\beta_{t}, w_{t}\right)$, where $\left(\beta_{t}, w_{t}\right) \in H_{0}^{1}(\Omega)^{2} \times H_{0}^{1}(\Omega)$ is the solution of

$$
\left\{\begin{array}{l}
a\left(\beta_{t}, \eta\right)+\left(\gamma_{t}, \nabla v-\eta\right)=(f, v)+\frac{t^{2}}{12}(\theta, \eta), \quad \forall(\eta, v) \in H_{0}^{1}(\Omega)^{2} \times H_{0}^{1}(\Omega), \\
\gamma_{t}=\frac{\kappa}{t^{2}}\left(\nabla w_{t}-\beta_{t}\right) .
\end{array}\right.
$$

We denote by $(\cdot, \cdot)_{t}$ the weighted $L^{2}(\Omega)^{2} \times L^{2}(\Omega)$ inner product in the right hand side of the first equation of (2.4) and by $|\cdot|_{t}$ its corresponding norm. Clearly $T_{t}$ is compact and selfadjoint with respect to $(\cdot, \cdot)_{t}$. Then, apart from $\mu=0$, its spectrum consists of a sequence of finite multiplicity real eigenvalues converging to zero. Note that $\lambda_{t}$ is an eigenvalue of (2.3) if and only if $\mu_{t}:=1 / \lambda_{t}$ is an eigenvalue of $T_{t}$ with the same multiplicity and corresponding eigenfunctions.

It is known (see [7]) that, when $t \rightarrow 0$, the solution of problem (2.4) converges to $\left(\beta_{0}, w_{0}\right) \in H_{0}^{1}(\Omega)^{2} \times H_{0}^{1}(\Omega)$ such that there exists $\gamma_{0} \in H_{0}(\operatorname{rot}, \Omega)^{\prime}$ satisfying

$$
\left\{\begin{array}{l}
a\left(\beta_{0}, \eta\right)+\left\langle\gamma_{0}, \nabla v-\eta\right\rangle=(f, v), \quad \forall(\eta, v) \in H_{0}^{1}(\Omega)^{2} \times H_{0}^{1}(\Omega), \\
\nabla w_{0}-\beta_{0}=0,
\end{array}\right.
$$


where $\langle\cdot, \cdot\rangle$ stands for the duality pairing in $H_{0}(\operatorname{rot}, \Omega):=\left\{\psi \in L^{2}(\Omega)^{2}: \operatorname{rot} \psi \in\right.$ $L^{2}(\Omega)$ and $\psi \cdot \tau=0$ on $\left.\partial \Omega\right\}$ ( $\tau$ being a unit vector tangent to $\partial \Omega$ ). This is a mixed formulation of the Kirchhoff model for the deflection of clamped thin plates:

$$
w_{0} \in H_{0}^{2}(\Omega) \quad \text { and } \quad \frac{E}{12\left(1-\nu^{2}\right)} \Delta^{2} w_{0}=f, \quad \text { in } \Omega .
$$

Moreover, defining $T_{0}(\theta, f):=\left(\beta_{0}, w_{0}\right)$ for $(\theta, f) \in L^{2}(\Omega)^{2} \times L^{2}(\Omega)$, we will show in the next section that for $0 \leq t \leq t_{\max }$

$$
\left\|\left(T_{t}-T_{0}\right)(\theta, f)\right\|_{H^{1}(\Omega)^{2} \times H^{1}(\Omega)} \leq C t|(\theta, f)|_{t} .
$$

In particular, since $|\cdot|_{t} \leq t_{\max }\|\cdot\|_{H^{1}(\Omega)^{2} \times H^{1}(\Omega)}$ for $t \in\left(0, t_{\max }\right)$, it follows that $\left.T_{t}\right|_{H^{1}(\Omega)^{2} \times H^{1}(\Omega)}$ converges to $\left.T_{0}\right|_{H^{1}(\Omega)^{2} \times H^{1}(\Omega)}$ in norm. Then, standard properties of separation of isolated parts of the spectrum (see for instance [14]) yield the following result:

Lemma 2.1. Let $\mu_{0}>0$ be an eigenvalue of $T_{0}$ of multiplicity $m$. Let $D$ be any disc in the complex plane centered at $\mu_{0}$ and containing no other element of the spectrum of $T_{0}$. Then, for $t$ small enough, $D$ contains exactly $m$ eigenvalues of $T_{t}$ (repeated according to their respective multiplicities). Consequently, each eigenvalue $\mu_{0}>0$ of $T_{0}$ is a limit of eigenvalues $\mu_{t}$ of $T_{t}$, as $t$ goes to zero.

Mixed finite elements for the load problem (2.4) have been introduced and analyzed in several papers (see for example $[6,8,10,15,16]$ ). The method that we will use here can be seen as the lowest degree member of the so-called MITC elements, which are based on relaxing the second equation of (2.3). In order to recall this method let us introduce some notation.

Assume that we have a family of triangulations $\left\{\mathcal{T}_{h}\right\}$ satisfying the usual minimum angle condition. The finite element space for the rotations consists of piecewise linear functions augmented in such a way that they have quadratic tangential components on the boundary of each element. Namely, for each $K \in \mathcal{T}_{h}$, let $n$ be a unit normal on $\partial K$ and define

$$
\mathcal{Q}(K):=\left\{\eta \in \mathcal{P}_{2}(K)^{2}:\left.\eta \cdot n\right|_{\ell} \in \mathcal{P}_{1}(\ell), \text { for each edge } \ell \text { of } K\right\} ;
$$

then, the finite element space for the rotations is defined by

$$
H_{h}:=\left\{\eta \in H_{0}^{1}(\Omega)^{2}:\left.\eta\right|_{K} \in \mathcal{Q}(K), \forall K \in \mathcal{T}_{h}\right\} .
$$

For the transverse displacements we take standard piecewise linear elements, namely,

$$
W_{h}:=\left\{v \in H_{0}^{1}(\Omega):\left.v\right|_{K} \in \mathcal{P}_{1}(K), \forall K \in \mathcal{T}_{h}\right\} .
$$

In order to define the method, we also need the reduction operator

$$
R: H^{1}(\Omega)^{2} \cap H_{0}(\operatorname{rot}, \Omega) \longrightarrow \Gamma_{h},
$$

where $\Gamma_{h}$ is the lowest order rotated Raviart-Thomas space, namely,

$$
\Gamma_{h}:=\left\{\psi \in H_{0}(\operatorname{rot}, \Omega):\left.\psi\right|_{K} \in \mathcal{P}_{0}^{2} \oplus\left(-x_{2}, x_{1}\right) \mathcal{P}_{0}, \forall K \in \mathcal{T}_{h}\right\},
$$

and $R$ is the operator locally defined for each $\psi \in H^{1}(\Omega)^{2}$ by (see $[7,17]$ )

$$
\int_{\ell} R \psi \cdot \tau=\int_{\ell} \psi \cdot \tau
$$


for every edge $\ell$ of the triangulation ( $\tau$ being a unit tangent vector along $\ell$ ). It is easy to see that the operator $R$ satisfies

$$
\int_{K} \operatorname{rot}(\psi-R \psi)=0, \quad \forall \psi \in H^{1}(\Omega)^{2},
$$

for any element $K \in \mathcal{T}_{h}$, and it is also known $([7,17])$ that

$$
\|\psi-R \psi\|_{0} \leq C h\|\psi\|_{1}
$$

here and hereafter $\|\cdot\|_{k}$ denotes the standard norm of $H^{k}(\Omega)$ or $H^{k}(\Omega)^{2}$ (which one will be obvious).

Now, the finite element approximate solution $\left(\beta_{t h}, w_{t h}\right) \in H_{h} \times W_{h}$ of the load problem (2.4) is defined by

$$
\left\{\begin{array}{l}
a\left(\beta_{t h}, \eta\right)+\left(\gamma_{t h}, \nabla v-R \eta\right)=(f, v)+\frac{t^{2}}{12}(\theta, \eta), \quad \forall(\eta, v) \in H_{h} \times W_{h}, \\
\gamma_{t h}=\frac{\kappa}{t^{2}}\left(\nabla w_{t h}-R \beta_{t h}\right) .
\end{array}\right.
$$

The method is nonconforming, since consistency terms arise because of the reduction operator. Existence and uniqueness of the solution of (2.10) follow easily (see [10]). As in the continuous case, we introduce the operator

$$
T_{t h}: L^{2}(\Omega)^{2} \times L^{2}(\Omega) \longrightarrow L^{2}(\Omega)^{2} \times L^{2}(\Omega)
$$

given by $T_{t h}(\theta, f):=\left(\beta_{t h}, w_{t h}\right) . T_{t h}$ is also selfadjoint with respect to $(\cdot, \cdot)_{t}$.

The corresponding eigenvalue problem reads

$$
\left\{\begin{array}{l}
a\left(\beta_{t h}, \eta\right)+\left(\gamma_{t h}, \nabla v-R \eta\right)=\lambda_{t h}\left[\left(w_{t h}, v\right)+\frac{t^{2}}{12}\left(\beta_{t h}, \eta\right)\right], \quad \forall(\eta, v) \in H_{h} \times W_{h}, \\
\gamma_{t h}=\frac{\kappa}{t^{2}}\left(\nabla w_{t h}-R \beta_{t h}\right) .
\end{array}\right.
$$

Once more $\lambda_{t h}$ is an eigenvalue of this problem if and only if $\mu_{t h}:=1 / \lambda_{t h}$ is a strictly positive eigenvalue of $T_{t h}$ with the same multiplicity and corresponding eigenfunctions.

For $t>0$ fixed, the spectral theory for compact operators in [3] can be directly applied to prove convergence of the eigenpairs of $T_{t h}$ to those of $T_{t}$. However, further considerations are needed to show that the error estimates do not deteriorate as $t$ becomes small.

To this goal we will make use of the following result, which means that optimal error estimates in the $H^{1}$ norm for the rotations and the transverse displacement hold for the approximations given by (2.10):

$$
\left\|\left(T_{t}-T_{t h}\right)(\theta, f)\right\|_{H^{1}(\Omega)^{2} \times H^{1}(\Omega)} \leq C h|(\theta, f)|_{t},
$$

with a constant $C$ independent of $t$ and $h$. This has been proved for instance in [10] for pure transversal loads (i.e., $\theta=0$ ), but the proofs therein extend trivially to our case.

As a consequence of (2.11), if $\mu_{t}$ is an eigenvalue of $T_{t}$ with multiplicity $m$, then exactly $m$ eigenvalues $\mu_{t h}^{(1)}, \ldots, \mu_{t h}^{(m)}$ of $T_{t h}$ (repeated according to their respective multiplicities) converge to $\mu_{t}$ as $h$ goes to zero (see [14]). The following theorem shows that, under mild assumptions, optimal $t$-independent error estimates in the $H^{1}$ norm are valid for the eigenfunctions: 
Theorem 2.1. Let $\mu_{t}$ be an eigenvalue of $T_{t}$ converging to a simple eigenvalue $\mu_{0}$ of $T_{0}$, as $t$ goes to zero. Let $\mu_{t h}$ be the eigenvalue of $T_{t h}$ that converges to $\mu_{t}$, as $h$ goes to zero. Let $\left(\beta_{t}, w_{t}\right)$ and $\left(\beta_{t h}, w_{t h}\right)$ be the corresponding eigenfunctions normalized in the same manner. Then, for $t$ and $h$ small enough,

$$
\left\|\left(\beta_{t}, w_{t}\right)-\left(\beta_{t h}, w_{t h}\right)\right\|_{H^{1}(\Omega)^{2} \times H^{1}(\Omega)} \leq C h,
$$

with a constant $C$ independent of $t$ and $h$.

Proof. For any fixed $t \in\left(0, t_{\max }\right),|\cdot|_{t} \leq t_{\max }\|\cdot\|_{H^{1}(\Omega)^{2} \times H^{1}(\Omega)}$. Hence, because of (2.11), $\left.T_{t h}\right|_{H^{1}(\Omega)^{2} \times H^{1}(\Omega)}$ converges to $\left.T_{t}\right|_{H^{1}(\Omega)^{2} \times H^{1}(\Omega)}$ in norm. Then (2.12) is a direct consequence of the estimate (2.11) and Theorem 7.1 in [3], with a constant $C$ depending on the constant in (2.11) (which is independent of $t$ ) and on the inverse of the distance from $\mu_{t}$ to the rest of the spectrum of $T_{t}$. Now, according to Lemma 2.1, (2.6) implies that for $t$ small enough this distance can be bounded below in terms of the distance from $\mu_{0}$ to the rest of the spectrum of $T_{0}$, which obviously does not depend on $t$.

In the next section we will prove the following higher order $L^{2}$ error estimate for the aproximate solution of the load problem (2.4):

$$
\left\|\left(T_{t}-T_{t h}\right)(\theta, f)\right\|_{L^{2}(\Omega)^{2} \times L^{2}(\Omega)} \leq C h^{2}|(\theta, f)|_{t},
$$

with a constant $C$ independent of $t$ and $h$. By using it we are able to prove a double order of convergence for the eigenvalues:

Theorem 2.2. Let $\mu_{t}$ and $\mu_{t h}$ be as in Theorem 2.1. Then, for $t$ and $h$ small enough,

$$
\left|\mu_{t}-\mu_{t h}\right| \leq C h^{2}
$$

with a constant $C$ independent of $t$ and $h$.

Proof. Let $\left(\beta_{t}, w_{t}\right)$ be an eigenfunction corresponding to $\mu_{t}$ normalized in $|\cdot|_{t}$. Since $T_{t}$ and $T_{t h}$ are selfadjoint with respect to $(\cdot, \cdot)_{t}$, we may apply Remark 7.5 in [3], which in our case reads

$$
\left|\mu_{t}-\mu_{t h}\right| \leq C\left[\left(\left(T_{t}-T_{t h}\right)\left(\beta_{t}, w_{t}\right),\left(\beta_{t}, w_{t}\right)\right)_{t}+\left|\left(T_{t}-T_{t h}\right)\left(\beta_{t}, w_{t}\right)\right|_{t}^{2}\right],
$$

with a constant $C$ depending only on the inverse of the distance from $\mu_{t}$ to the rest of the spectrum of $T_{t}$. By repeating the arguments in the proof of Theorem 2.1 we observe that, for $t$ small enough, this constant can be chosen independent of $t$. Thus, since $|\cdot|_{t} \leq t_{\max }\|\cdot\|_{L^{2}(\Omega)^{2} \times L^{2}(\Omega)}$, by using estimate (2.13) in (2.14) we conclude the proof.

Another consequence of estimate (2.13) is a double order of convergence in the $L^{2}$ norm for the eigenfunctions:

Theorem 2.3. Let $\mu_{t}, \mu_{t h},\left(\beta_{t}, w_{t}\right)$ and $\left(\beta_{t h}, w_{t h}\right)$ be as in Theorem 2.1. Then, for $t$ and $h$ small enough,

$$
\left\|\left(\beta_{t}, w_{t}\right)-\left(\beta_{t h}, w_{t h}\right)\right\|_{L^{2}(\Omega)^{2} \times L^{2}(\Omega)} \leq C h^{2},
$$

with a constant $C$ independent of $t$ and $h$.

Proof. Since $|\cdot|_{t} \leq t_{\max }\|\cdot\|_{L^{2}(\Omega)^{2} \times L^{2}(\Omega)}$, the arguments in the proof of Theorem 2.1 can be repeated using $\|\cdot\|_{L^{2}(\Omega)^{2} \times L^{2}(\Omega)}$ instead of $\|\cdot\|_{H^{1}(\Omega)^{2} \times H^{1}(\Omega)}$ and estimate (2.13) instead of estimate (2.11). 
The three theorems of this section are stated for those eigenvalues of ReissnerMindlin equations converging to simple eigenvalues of the Kirchhoff model. A multiple eigenvalue of the latter arises usually because of symmetries of the geometry of the plate; in such a case, the eigenvalue of the former converging to it has the same multiplicity. The proofs of these theorems extend trivially to cover this case.

Instead, if the Kirchhoff equations had a multiple eigenvalue not due to symmetry reasons, it could split into different eigenvalues in the Reissner-Mindlin model. In this case, the proofs of the theorems above do not provide estimates independent of the thickness. In fact, the constants therein blow up as the distance between the Reissner-Mindlin eigenvalues becomes smaller.

For conforming methods, the minimum-maximum principles yield estimates not involving this distance (see, for instance, Section 8 of [3]). However, to the best of our knowledge, estimates of this kind have not been proved for non-conforming methods like ours. Nevertheless, the numerical experiments in Section 4 show that such estimates also hold in this case for our method.

\section{Proofs}

The optimal spectral convergence results in the previous section rely on properties (2.6), (2.11) and (2.13). The proof of the first one is standard, but we include it for the sake of completeness. The second one is an $H^{1}$ norm estimate for the load problem including shear loads, and its proof is an immediate extension of those in $[8,10,16]$. On the other hand, property $(2.13)$, regarding the approximation with optimal order in the $L^{2}$ norm, was not previously known. Similar $L^{2}$ estimates have been proved for higher order methods (see $[8,15]$ ), but with proofs relying on arguments which do not apply to the lowest order case we are considering.

In what follows we will make use of the known a priori estimates for the solutions of problems (2.4) and (2.5) (see for instance [2]):

$$
\begin{aligned}
\left\|\beta_{t}\right\|_{2}+\left\|w_{t}\right\|_{2}+\left\|\gamma_{t}\right\|_{0}+t\left\|\gamma_{t}\right\|_{1} & \leq C\left(t^{2}\|\theta\|_{0}+\|f\|_{0}\right) \\
& \leq C|(\theta, f)|_{t},
\end{aligned}
$$

valid for $0 \leq t \leq t_{\max }$, with a constant $C$ independent of $t$.

We begin with the proof of property (2.6).

Lemma 3.1. There exists a constant $C$, independent of $t$, such that

$$
\left\|\left(T_{t}-T_{0}\right)(\theta, f)\right\|_{H^{1}(\Omega)^{2} \times H^{1}(\Omega)} \leq C t|(\theta, f)|_{t},
$$

for all $(\theta, f) \in L^{2}(\Omega)^{2} \times L^{2}(\Omega)$.

Proof. Subtracting (2.5) from (2.4), we have

$$
\left\{\begin{array}{l}
a\left(\beta_{t}-\beta_{0}, \eta\right)+\left(\gamma_{t}-\gamma_{0}, \nabla v-\eta\right)=\frac{t^{2}}{12}(\theta, \eta), \quad \forall(\eta, v) \in H_{0}^{1}(\Omega)^{2} \times H_{0}^{1}(\Omega), \\
\gamma_{t}=\frac{\kappa}{t^{2}}\left[\nabla\left(w_{t}-w_{0}\right)-\left(\beta_{t}-\beta_{0}\right)\right],
\end{array}\right.
$$

and taking $\eta=\beta_{t}-\beta_{0}$ and $v=w_{t}-w_{0}$, we obtain

$$
a\left(\beta_{t}-\beta_{0}, \beta_{t}-\beta_{0}\right)=\frac{t^{2}}{12}\left(\theta, \beta_{t}-\beta_{0}\right)-\frac{t^{2}}{\kappa}\left(\gamma_{t}-\gamma_{0}, \gamma_{t}\right) .
$$


Now, using the coerciveness of $a$ and the $a$ priori estimate (3.1) for $\left\|\gamma_{t}\right\|_{0}$ and $\left\|\gamma_{0}\right\|_{0}$, we have

$$
\begin{aligned}
\left\|\beta_{t}-\beta_{0}\right\|_{1}^{2} & \leq C t^{2}\|\theta\|_{0}\left\|\beta_{t}-\beta_{0}\right\|_{0}+C t^{2}\left(\left\|\gamma_{t}\right\|_{0}+\left\|\gamma_{0}\right\|_{0}\right)\left\|\gamma_{t}\right\|_{0} \\
& \leq C t|(\theta, f)|_{t}\left\|\beta_{t}-\beta_{0}\right\|_{0}+C t^{2}|(\theta, f)|_{t}^{2},
\end{aligned}
$$

and therefore

$$
\left\|\beta_{t}-\beta_{0}\right\|_{1} \leq C t|(\theta, f)|_{t}
$$

Finally, observe that

$$
\nabla\left(w_{t}-w_{0}\right)=\left(\beta_{t}-\beta_{0}\right)+\frac{t^{2}}{\kappa} \gamma_{t},
$$

and so, using again the a priori estimate (3.1) for $\left\|\gamma_{t}\right\|_{0}$, we obtain

$$
\left\|w_{t}-w_{0}\right\|_{1} \leq C\left(\left\|\beta_{t}-\beta_{0}\right\|_{0}+t^{2}|(\theta, f)|_{t}\right)
$$

which together with (3.2) allow us to conclude the proof.

The arguments to prove the remaining properties are based on the fact that there exists an operator

$$
\Pi: H_{0}^{1}(\Omega)^{2} \times\left[H_{0}^{1}(\Omega) \cap H^{2}(\Omega)\right] \longrightarrow H_{h} \times W_{h}
$$

such that, if $(\hat{\eta}, \hat{w}):=\Pi(\eta, w)$, then

$$
R(\nabla w-\eta)=\nabla \hat{w}-R \hat{\eta}
$$

( $R$ being the reduction operator satisfying $(2.7),(2.8)$ and $(2.9))$ and

$$
\|\eta-\hat{\eta}\|_{1} \leq C h\|\eta\|_{2} .
$$

The construction of such an operator is based on known properties of the spaces $H_{h}, W_{h}$ and $\Gamma_{h}$ (see [10] for details). It is worth observing that (3.3) corresponds to a commutative diagram property, usual in the analysis of mixed methods. In fact, introducing the operators $B$ and $B_{h}$ such that $B(\eta, v):=\nabla v-\eta$, for $(\eta, v) \in$ $H_{0}^{1}(\Omega)^{2} \times H_{0}^{1}(\Omega)$, and $B_{h}(\eta, v):=\nabla v-R \eta$, for $(\eta, v) \in H_{h} \times W_{h}$, that property can be summarized in the following commutative diagram:

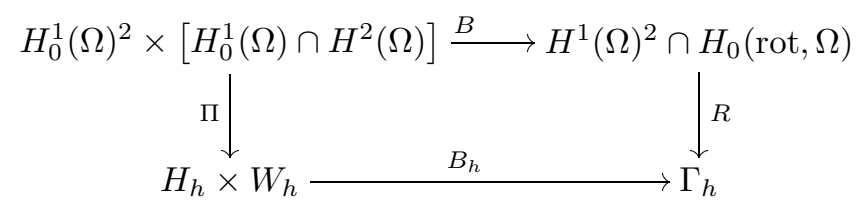

Note that, if $R$ were an $L^{2}$ projection, this commutative diagram would correspond to Fortin's well-known property (which in its turn implies that an inf-sup condition, analogous to that proved in [7] for the continuous problem, holds for the discrete case). Of course, $R$ is not an $L^{2}$ projection, and so the commutative diagram property given above can be seen as a generalization of Fortin's one. In fact, optimal error estimates in $H^{1}$ for the rotations and the transverse displacement yielding (2.11) follow from this generalized property:

Lemma 3.2. There exists a constant $C$, independent of $t$ and $h$, such that

$$
\left\|\left(T_{t}-T_{t h}\right)(\theta, f)\right\|_{H^{1}(\Omega)^{2} \times H^{1}(\Omega)} \leq C h|(\theta, f)|_{t},
$$

for all $(\theta, f) \in L^{2}(\Omega)^{2} \times L^{2}(\Omega)$. 
Proof. Arguments identical to those in [10], combined with the a priori estimate (3.1), yield in our case

$$
\begin{aligned}
\left\|\beta_{t}-\beta_{t h}\right\|_{1}+t\left\|\gamma_{t}-\gamma_{t h}\right\|_{0} & \leq C h\left(\left\|\beta_{t}\right\|_{2}+t\left\|\gamma_{t}\right\|_{1}+\left\|\gamma_{t}\right\|_{0}\right) \\
& \leq C h|(\theta, f)|_{t},
\end{aligned}
$$

and, as a consequence,

$$
\begin{aligned}
\left\|w_{t}-w_{t h}\right\|_{1} & \leq C h\left(\left\|\beta_{t}\right\|_{2}+t\left\|\gamma_{t}\right\|_{1}+\left\|\gamma_{t}\right\|_{0}\right) \\
& \leq C h|(\theta, f)|_{t},
\end{aligned}
$$

therefore concluding the proof.

We will use a duality argument to show that, under the same conditions, optimal $L^{2}$ error estimates for the rotations and the transverse displacement also hold. First we will prove a lemma which will be useful to bound the consistency terms arising from the reduced integration of the shear strain.

For $\psi \in H_{0}^{1}(\Omega)^{2}$, we denote by $\psi_{I} \in H_{0}^{1}(\Omega)^{2}$ a piecewise linear average interpolant as defined in $[9,18]$, satisfying

$$
\left\|\psi_{I}\right\|_{1} \leq C\|\psi\|_{1}
$$

and

$$
\left\|\psi-\psi_{I}\right\|_{1} \leq C h\|\psi\|_{2}
$$

The following estimate holds:

Lemma 3.3. Given $\zeta \in H(\operatorname{div}, \Omega)$ and $\psi \in H_{0}^{1}(\Omega)^{2}$, we have

$$
\left|\left(\zeta, \psi_{I}-R \psi_{I}\right)\right| \leq C h^{2}\|\operatorname{div} \zeta\|_{0}\|\psi\|_{1} .
$$

Proof. From property (2.8) it follows that

$$
\operatorname{rot} R \psi_{I}=P\left(\operatorname{rot} \psi_{I}\right),
$$

where $P$ is the $L^{2}$ projection onto the piecewise constant functions. Now, since $\left.\left(\operatorname{rot} \psi_{I}\right)\right|_{K} \in \mathcal{P}_{0}$, then $\left(\operatorname{rot} \psi_{I}\right)=P\left(\operatorname{rot} \psi_{I}\right)$. Hence $\operatorname{rot}\left(\psi_{I}-R \psi_{I}\right)=0$, and so there exists $r \in H^{1}(\Omega)$ such that

$$
\nabla r=\psi_{I}-R \psi_{I}
$$

actually, we can take $r \in H_{0}^{1}(\Omega)$ because the tangential component of $\left(\psi_{I}-R \psi_{I}\right)$ vanishes on $\partial \Omega$. Then, we have

$$
\left|\left(\zeta, \psi_{I}-R \psi_{I}\right)\right|=|(\zeta, \nabla r)|=|(\operatorname{div} \zeta, r)| \leq\|\operatorname{div} \zeta\|_{0}\|r\|_{0} .
$$

On the other hand, from property (2.7) defining $R$ it follows that, for every edge $\ell$ with end points $A$ and $B$, we have

$$
r(A)-r(B)=\int_{\ell} \nabla r \cdot \tau=\int_{\ell}\left(\psi_{I}-R \psi_{I}\right) \cdot \tau=0 .
$$

Therefore, since $r$ vanishes on $\partial \Omega$ we conclude that $r$ vanishes at all the nodes of the triangulation. Hence, since $\left.r\right|_{K} \in \mathcal{P}_{2}$, a standard scaling argument on each element $K$ yields

$$
\|r\|_{0} \leq C h\|\nabla r\|_{0}
$$

which together with (2.9), (3.6), (3.8) and (3.9) allows us to conclude the proof. 
Finally, we will prove the main result of this section, property (2.13), concerning $L^{2}$ error estimates optimal in order and regularity. Let us remark that this result completes the analysis of the MITC elements carried out in $[8,15]$ for the higher order methods. (To simplify the notation, in this lemma we will drop the subscript $t$ from $\beta_{t}, w_{t}, \gamma_{t}$ and from their discrete approximations.)

Lemma 3.4. Given $(\theta, f) \in L^{2}(\Omega)^{2} \times L^{2}(\Omega)$, let $(\beta, w)=T_{t}(\theta, f)$ and $\left(\beta_{h}, w_{h}\right)=$ $T_{t h}(\theta, f)$. Then there exists a constant $C$, independent of $t$ and $h$, such that

$$
\left\|\beta-\beta_{h}\right\|_{0}+\left\|w-w_{h}\right\|_{0} \leq C h^{2}|(\theta, f)|_{t}
$$

or, equivalently,

$$
\left\|\left(T_{t}-T_{t h}\right)(\theta, f)\right\|_{L^{2}(\Omega)^{2} \times L^{2}(\Omega)} \leq C h^{2}|(\theta, f)|_{t} .
$$

Proof. Subtracting (2.10) from (2.4), we obtain the error equation

$$
a\left(\beta-\beta_{h}, \eta\right)+\left(\gamma-\gamma_{h}, \nabla v-R \eta\right)=(\gamma, \eta-R \eta), \quad \forall(\eta, v) \in H_{h} \times W_{h},
$$

with $\gamma=\frac{\kappa}{t^{2}}(\nabla w-\beta)$ and $\gamma_{h}=\frac{\kappa}{t^{2}}\left(\nabla w_{h}-R \beta_{h}\right)$.

We will use a duality argument: let $(\varphi, u) \in H_{0}^{1}(\Omega)^{2} \times H_{0}^{1}(\Omega)$ be the solution of

$$
\left\{\begin{array}{l}
a(\eta, \varphi)+(\nabla v-\eta, \delta)=\left(v, w-w_{h}\right)+\left(\eta, \beta-\beta_{h}\right), \quad \forall(\eta, v) \in H_{0}^{1}(\Omega)^{2} \times H_{0}^{1}(\Omega), \\
\delta=\frac{\kappa}{t^{2}}(\nabla u-\varphi) .
\end{array}\right.
$$

An a priori estimate analogous to (3.1) is valid for this problem, namely,

$$
\|\varphi\|_{2}+\|u\|_{2}+\|\delta\|_{0}+t\|\delta\|_{1} \leq C\left(\left\|\beta-\beta_{h}\right\|_{0}+\left\|w-w_{h}\right\|_{0}\right),
$$

and by taking $\eta=0$ in (3.11) we have

$$
-\operatorname{div} \delta=w-w_{h} .
$$

By taking $v=w-w_{h}$ and $\eta=\beta-\beta_{h}$ in the dual problem (3.11) we have

$$
\begin{aligned}
\| w- & w_{h}\left\|_{0}^{2}+\right\| \beta-\beta_{h} \|_{0}^{2} \\
& =a\left(\beta-\beta_{h}, \varphi\right)+\left(\nabla\left(w-w_{h}\right)-\left(\beta-R \beta_{h}\right), \delta\right)+\left(\beta_{h}-R \beta_{h}, \delta\right) \\
& =a\left(\beta-\beta_{h}, \varphi\right)+\frac{t^{2}}{\kappa}\left(\gamma-\gamma_{h}, \delta\right)+\left(\beta_{h}-R \beta_{h}, \delta\right),
\end{aligned}
$$

and using the error equation (3.10) with $(\eta, v)=(\hat{\varphi}, \hat{u}):=\Pi(\varphi, u)$ we obtain

$$
\begin{aligned}
\| w- & w_{h}\left\|_{0}^{2}+\right\| \beta-\beta_{h} \|_{0}^{2} \\
= & a\left(\beta-\beta_{h}, \varphi-\hat{\varphi}\right)+\frac{t^{2}}{\kappa}\left(\gamma-\gamma_{h}, \delta\right)-\left(\gamma-\gamma_{h}, \nabla \hat{u}-R \hat{\varphi}\right) \\
& +\left(\beta_{h}-R \beta_{h}, \delta\right)+(\gamma, \hat{\varphi}-R \hat{\varphi}) . \\
= & a\left(\beta-\beta_{h}, \varphi-\hat{\varphi}\right)+\frac{t^{2}}{\kappa}\left(\gamma-\gamma_{h}, \delta-R \delta\right) \\
& +\left(\beta_{h}-R \beta_{h}, \delta\right)+(\gamma, \hat{\varphi}-R \hat{\varphi}),
\end{aligned}
$$


where we have used the commutative diagram property $(3.3), R \delta=\frac{\kappa}{t^{2}}(\nabla \hat{u}-R \hat{\varphi})$, for the last equality. Now it only remains to estimate the four terms in the last expression.

The first two can be easily bounded. In fact, using the error estimates (3.4) and (3.5) and the a priori estimate (3.12) we have

$$
\begin{aligned}
\left|a\left(\beta-\beta_{h}, \varphi-\hat{\varphi}\right)\right| & \leq C\left\|\beta-\beta_{h}\right\|_{1}\|\varphi-\hat{\varphi}\|_{1} \leq C\left\|\beta-\beta_{h}\right\|_{1} h\|\varphi\|_{2} \\
& \leq C h^{2}|(\theta, f)|_{t}\left(\left\|\beta-\beta_{h}\right\|_{0}+\left\|w-w_{h}\right\|_{0}\right)
\end{aligned}
$$

and, from (2.9), (3.5) and (3.12),

$$
\begin{aligned}
& \frac{t^{2}}{\kappa}\left|\left(\gamma-\gamma_{h}, \delta-R \delta\right)\right| \\
& \quad \leq C t^{2}\left\|\gamma-\gamma_{h}\right\|_{0}\|\delta-R \delta\|_{0} \leq C t^{2}\left\|\gamma-\gamma_{h}\right\|_{0} h\|\delta\|_{1} \\
& \quad \leq C h^{2}|(\theta, f)|_{t}\left(\left\|\beta-\beta_{h}\right\|_{0}+\left\|w-w_{h}\right\|_{0}\right) .
\end{aligned}
$$

For the third term in (3.14) we have

$$
\left(\beta_{h}-R \beta_{h}, \delta\right)=\left(\left(\beta_{h}-\beta_{I}\right)-R\left(\beta_{h}-\beta_{I}\right), \delta\right)+\left(\beta_{I}-R \beta_{I}, \delta\right) .
$$

Now, using successively (2.9), (3.5), (3.7), (3.12) and (3.1), we obtain

$$
\begin{aligned}
& \left|\left(\left(\beta_{h}-\beta_{I}\right)-R\left(\beta_{h}-\beta_{I}\right), \delta\right)\right| \\
& \quad \leq C\|\delta\|_{0} h\left\|\beta_{h}-\beta_{I}\right\|_{1} \leq C\|\delta\|_{0} h\left(\left\|\beta_{h}-\beta\right\|_{1}+\left\|\beta-\beta_{I}\right\|_{1}\right) \\
& \quad \leq C h^{2}\left(\left\|\beta-\beta_{h}\right\|_{0}+\left\|w-w_{h}\right\|_{0}\right)|(\theta, f)|_{t},
\end{aligned}
$$

whereas by using Lemma 3.3, (3.13) and (3.1) we have

$$
\begin{aligned}
\left|\left(\beta_{I}-R \beta_{I}, \delta\right)\right| & \leq C h^{2}\|\beta\|_{1}\|\operatorname{div} \delta\|_{0} \\
& \leq C h^{2}|(\theta, f)|_{t}\left\|w-w_{h}\right\|_{0}
\end{aligned}
$$

Therefore

$$
\left|\left(\beta_{h}-R \beta_{h}, \delta\right)\right| \leq C h^{2}|(\theta, f)|_{t}\left(\left\|\beta-\beta_{h}\right\|_{0}+\left\|w-w_{h}\right\|_{0}\right) .
$$

The last term in (3.14) can be bounded in an almost identical way, by using (3.4) to estimate $\|\hat{\varphi}-\varphi\|_{1}$ and the fact that

$$
-\operatorname{div} \gamma_{t}=f, \quad \text { in } \Omega
$$

which follows by taking $\eta=0$ in problem (2.4) and (2.5).

By so doing we obtain

$$
|(\gamma, \hat{\varphi}-R \hat{\varphi})| \leq C h^{2}|(\theta, f)|_{t}\left(\left\|\beta-\beta_{h}\right\|_{0}+\left\|w-w_{h}\right\|_{0}\right) .
$$

Finally, from (3.14), (3.15), (3.16), (3.17) and (3.18), simple algebra allows us to conclude the proof. 


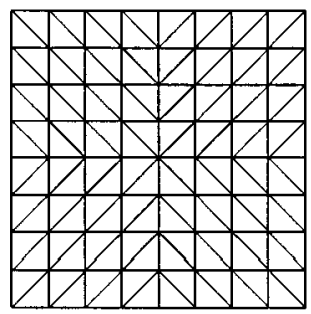

Figure 1. Finite element mesh for the square plate: $N=8$

\section{NUMERICAL EXPERIMENTS}

In this section we summarize the numerical experimentation carried out with our method. The aim of these computations was two-fold: to study the performance of the method and to discuss the pertinence of the assumptions made in Section 2 to prove error estimates.

To validate our codes and to test the effectiveness of the method to deal with different boundary conditions, we have first considered a typical benchmark problem: the computation of the lowest frequency vibration modes of a square plate. We have applied our codes to thin and moderately thick plates and compared the results with those reported in [1], in which the same problems were treated by means of a method introduced by Huang and Hinton in [12] that is based on biquadratic rectangular finite elements with enhanced shear interpolation.

We have considered a square plate of side length $L$ and two thickness-to-span $(t / L)$ ratios of 0.1 (moderately thick) and 0.01 (thin). In each case we have also considered four different types of boundary conditions:

- a clamped plate (as described in Section 2);

- a hard simply supported plate (i.e., with transversal displacements and rotations tangential to the boundary, both vanishing on each edge);

- a plate with mixed boundary conditions (with two opposite edges being hard simply supported and the other two clamped);

- a plate with a free edge (with three clamped edges and the fourth being free, i.e., no constraints either on the transversal displacements or on the rotations along this edge).

We denote each case by C-C-C-C, S-S-S-S, S-C-S-C and C-C-C-F, respectively (C stands for clamped, $\mathrm{S}$ for hard simply supported and $\mathrm{F}$ for free edges).

We have used succesive refinements of a uniform mesh like that in Figure 1, the refinement parameter $N$ being the number of element edges on each side of the square (hence, $h=\sqrt{2} L / N$ ).

We have applied our codes to the original unscaled problems analogous to (2.1). Thus we have computed approximations of the free vibration angular frequencies $\omega_{t}=t \sqrt{\lambda_{t} / \rho}$. In order to compare our results with those in [1] we present the computed frequencies $\omega_{m n}^{h}$ in the following non-dimensional form:

$$
\widehat{\omega}_{m n}:=\omega_{m n}^{h} L\left(\frac{2(1+\nu) \rho}{E}\right)^{1 / 2},
$$

$m$ and $n$ being the numbers of half-waves occurring in the modes shapes in the $x$ and $y$ directions, respectively. 
TABLE 1. Lowest non-dimensional vibration frequencies for moderately thick square plates: $t / L=0.1$

\begin{tabular}{|c||c||c|c|c||c||c|}
\hline Bound. cond. & Mode & $N=10$ & $N=20$ & $N=40$ & $\mathrm{H}-\mathrm{H}$ & $\mathrm{D}-\mathrm{R}$ \\
\hline C-C-C-C & $\widehat{\omega}_{11}$ & 1.5947 & 1.5921 & 1.5913 & 1.591 & 1.594 \\
& $\widehat{\omega}_{21}$ & 3.1181 & 3.0595 & 3.0441 & 3.039 & 3.046 \\
& $\widehat{\omega}_{12}$ & 3.1181 & 3.0595 & 3.0441 & 3.039 & 3.046 \\
& $\widehat{\omega}_{22}$ & 4.4477 & 4.3106 & 4.2746 & 4.263 & 4.285 \\
\hline S-S-S-S & $\widehat{\omega}_{11}$ & 0.9384 & 0.9323 & 0.9308 & 0.930 & 0.930 \\
& $\widehat{\omega}_{21}$ & 2.2893 & 2.2366 & 2.2236 & 2.219 & 2.219 \\
& $\widehat{\omega}_{12}$ & 2.2893 & 2.2366 & 2.2236 & 2.219 & 2.219 \\
& $\widehat{\omega}_{22}$ & 3.5657 & 3.4450 & 3.4153 & 3.405 & 3.406 \\
\hline S-C-S-C & $\widehat{\omega}_{11}$ & 1.3060 & 1.3016 & 1.3005 & 1.300 & 1.302 \\
& $\widehat{\omega}_{21}$ & 2.4664 & 2.4120 & 2.3984 & 2.394 & 2.398 \\
& $\widehat{\omega}_{12}$ & 2.9617 & 2.9043 & 2.8895 & 2.885 & 2.888 \\
& $\widehat{\omega}_{22}$ & 4.0126 & 3.8830 & 3.8500 & 3.839 & 3.852 \\
\hline C-C-C-F & $\widehat{\omega}_{\frac{1}{2} 1} 1$ & 1.0959 & 1.0848 & 1.0814 & 1.081 & 1.089 \\
& $\widehat{\omega}_{\frac{3}{2} 1}$ & 1.7759 & 1.7525 & 1.7454 & 1.744 & 1.758 \\
& $\widehat{\omega}_{\frac{1}{2} 2}$ & 2.7413 & 2.6787 & 2.6612 & 2.657 & 2.673 \\
& $\widehat{\omega}_{\frac{5}{2} 1}$ & 3.3186 & 3.2282 & 3.2035 & 3.197 & 3.216 \\
\hline
\end{tabular}

Tables 1 and 2 show the four lowest vibration frequencies computed by our method with three different meshes $(N=10,20,40)$ and each set of boundary conditions for each thickness-to-span ratio $(t / L=0.1$ and $t / L=0.01$, respectively). Each table includes the results obtained by Huang and Hinton's method in [12] (column H-H) and also by an analytical approximation obtained by Dawe and Roufaeil in [11] (column D-R), both as reported in [1]. In every case we have used a Poisson ratio $\nu=0.3$ and different correction factors depending on the boundary conditions, but always the same as those used in [1] to allow for the comparison: $k=0.8601$ for C-C-C-C and C-C-C-F, $k=0.8333$ for S-S-S-S and $k=0.822$ for S$\mathrm{C}-\mathrm{S}-\mathrm{C}$. The reported non-dimensional frequencies are independent of the remaining geometrical and physical parameters, except for the thickness-to-span ratio.

Both tables show that the method can be safely used for any of these boundary conditions and for thin as well as moderately thick plates.

The goal of our second experiment was to test if the hypothesis on the uniform separation of the spectrum assumed throughout Section 2 is actually necessary. For any convex clamped plate, according to Theorem 2.2, the convergence for each eigenvalue is quadratic. However, the constants in the error estimates arising in its proof blow up with the inverse of the distance from the approximated eigenvalue to the rest of the spectrum.

In order to test if this assumption is essential or if it only arises because of the techniques used to prove the theorems, we have considered a clamped rectangular steel plate with two very close vibration frequencies, but not exactly coincident. We have chosen the following values of the physical and geometric parameters: side lengths: $2 \mathrm{~m}$ and $1 \mathrm{~m}$; Young modulus: $E=1.43 \times 10^{11} \mathrm{~Pa}$; Poisson ratio: $\nu=0.35$; density: $\rho=7.7 \times 10^{3} \mathrm{Kg}$; and correction factor $k=5 / 6$. 
TABLE 2. Lowest non-dimensional vibration frequencies for thin square plates: $t / L=0.01$

\begin{tabular}{|c||c||c|c|c||c||c|}
\hline Bound. cond. & Mode & $N=10$ & $N=20$ & $N=40$ & H-H & D-R \\
\hline C-C-C-C & $\widehat{\omega}_{11}$ & 0.1754 & 0.1754 & 0.1754 & 0.1754 & 0.1754 \\
& $\widehat{\omega}_{21}$ & 0.3668 & 0.3599 & 0.3580 & 0.3574 & 0.3576 \\
& $\widehat{\omega}_{12}$ & 0.3668 & 0.3599 & 0.3580 & 0.3574 & 0.3576 \\
& $\widehat{\omega}_{22}$ & 0.5487 & 0.5323 & 0.5279 & 0.5264 & 0.5274 \\
\hline S-S-S-S & $\widehat{\omega}_{11}$ & 0.0972 & 0.0965 & 0.0963 & 0.0963 & 0.0963 \\
& $\widehat{\omega}_{21}$ & 0.2486 & 0.2426 & 0.2411 & 0.2406 & 0.2406 \\
& $\widehat{\omega}_{12}$ & 0.2486 & 0.2426 & 0.2411 & 0.2406 & 0.2406 \\
& $\widehat{\omega}_{22}$ & 0.4035 & 0.3893 & 0.3859 & 0.3847 & 0.3848 \\
\hline S-C-S-C & $\widehat{\omega}_{11}$ & 0.1417 & 0.1413 & 0.1412 & 0.1411 & 0.1411 \\
& $\widehat{\omega}_{21}$ & 0.2748 & 0.2688 & 0.2673 & 0.2668 & 0.2668 \\
& $\widehat{\omega}_{12}$ & 0.3474 & 0.3402 & 0.3383 & 0.3377 & 0.3377 \\
& $\widehat{\omega}_{22}$ & 0.4814 & 0.4657 & 0.4617 & 0.4604 & 0.4608 \\
\hline C-C-C-F & $\widehat{\omega}_{\frac{1}{2} 1}$ & 0.1182 & 0.1170 & 0.1167 & 0.1166 & 0.1171 \\
& $\widehat{\omega}_{\frac{3}{2} 1}$ & 0.1977 & 0.1956 & 0.1950 & 0.1949 & 0.1951 \\
& $\widehat{\omega}_{\frac{1}{2} 2}$ & 0.3193 & 0.3109 & 0.3086 & 0.3080 & 0.3093 \\
& $\widehat{\omega}_{\frac{5}{2} 1} 1$ & 0.3874 & 0.3771 & 0.3744 & 0.3736 & 0.3740 \\
\hline
\end{tabular}

We have considered again two plates of different thickness: one moderately thick $(t=0.1 \mathrm{~m})$ and the other thin $(t=0.01 \mathrm{~m})$. For each of them we have computed the lowest frequency vibration modes with different successively refined uniform meshes analogous to that in Figure 1, the parameter $N$ being now the number of edge elements on the shortest side of the plate.

We have observed that the relative error of the approximated angular frequencies $\omega_{m n}^{h}$ roughly behaves like

$$
\frac{\omega_{m n}^{h}-\omega_{m n}}{\omega_{m n}} \approx C_{m n} h^{\alpha},
$$

with an order of convergence $\alpha$ very close to 2 and constants $C_{m n}$ which depend on the numbers of half-waves $m$ and $n$, but which are almost independent of the thickness of the plate. We have also observed that these constants remain remarkably stable even for very close eigenvalues.

Then, for each mode, we have estimated the exact vibration frequencies $\omega_{m n}$, the value of the constants $C_{m n}$ and the order of convergence $\alpha$ by means of a least square fitting of the model

$$
\omega_{m n}^{h} \approx \omega_{m n}\left(1+C_{m n} h^{\alpha}\right)
$$

to the approximate frequencies computed on highly refined meshes $(N=16,32$, $48,64,80)$.

We summarize our results in Table 3 for the moderately thick plate and in Table 4 for the thin one.

It can be observed that the fourth and the fifth eigenvalues in both tables are very close (approximately 1\% of difference). However the corresponding constants $C_{m n}$ are not larger than those for other eigenvalues. This fact suggests that the 
TABLE 3. Lowest vibration frequencies (in $\mathrm{rad} / \mathrm{s}$ ) of a clamped moderately thick rectangular plate: $2 \mathrm{~m} \times 1 \mathrm{~m}, t=0.1 \mathrm{~m}$

\begin{tabular}{|c||c|c|c||c||c||c|}
\hline Mode & $N=8$ & $N=16$ & $N=32$ & $\alpha$ & $\omega_{m n}$ & $C_{m n}$ \\
\hline$\omega_{11}^{h}$ & 3035.212 & 3025.827 & 3023.214 & 1.97 & 3022.317 & 0.071 \\
$\omega_{21}^{h}$ & 3918.755 & 3875.817 & 3864.488 & 1.98 & 3860.631 & 0.242 \\
$\omega_{31}^{h}$ & 5441.309 & 5351.958 & 5328.053 & 1.97 & 5319.841 & 0.365 \\
$\omega_{12}^{h}$ & 7536.399 & 7333.836 & 7280.257 & 1.98 & 7262.144 & 0.616 \\
$\omega_{41}^{h}$ & 7630.812 & 7399.422 & 7338.489 & 1.98 & 7317.795 & 0.689 \\
$\omega_{22}^{h}$ & 8340.381 & 8076.061 & 8006.128 & 1.98 & 7982.469 & 0.731 \\
$\omega_{32}^{h}$ & 9673.715 & 9326.597 & 9233.320 & 1.97 & 9201.449 & 0.830 \\
\hline
\end{tabular}

TABLE 4. Lowest vibration frequencies (in $\mathrm{rad} / \mathrm{s}$ ) of a clamped thin rectangular plate: $2 \mathrm{~m} \times 1 \mathrm{~m}, t=0.01 \mathrm{~m}$

\begin{tabular}{|c||c|c|c||c||c||c|}
\hline Mode & $N=8$ & $N=16$ & $N=32$ & $\alpha$ & $\omega_{m n}$ & $C_{m n}$ \\
\hline$\omega_{11}^{h}$ & 328.760 & 327.663 & 327.347 & 1.99 & 327.240 & 0.086 \\
$\omega_{21}^{h}$ & 429.627 & 425.210 & 424.050 & 2.00 & 423.665 & 0.239 \\
$\omega_{31}^{h}$ & 607.831 & 598.992 & 596.605 & 1.98 & 595.794 & 0.336 \\
$\omega_{41}^{h}$ & 878.302 & 851.895 & 844.852 & 1.98 & 842.468 & 0.702 \\
$\omega_{12}^{h}$ & 888.126 & 860.941 & 853.510 & 1.98 & 850.980 & 0.727 \\
$\omega_{22}^{h}$ & 991.486 & 957.454 & 948.250 & 1.98 & 945.136 & 0.819 \\
$\omega_{32}^{h}$ & 1162.198 & 1121.967 & 1110.801 & 1.97 & 1106.970 & 0.828 \\
\hline
\end{tabular}

assumption on the separation of the spectrum made in Section 2 is not really necessary.

Finally, we have made a stringent test of this hypothesis and, at the same time, of the stability of the method as $t$ goes to zero. The eigenvalues of Kirchhoff equations for a rectangular plate with simply supported boundary conditions are exactly known:

$$
\lambda_{0}^{m n}:=\frac{E \pi^{4}}{12\left(1-\nu^{2}\right)}\left(\frac{m^{2}}{a^{2}}+\frac{n^{2}}{b^{2}}\right)^{2}, \quad m, n=1,2,3, \ldots,
$$

where $a$ and $b$ are the side lengths of the plate. If we consider the same lengths as in the previous experiment $(a=2 \mathrm{~m}, b=1 \mathrm{~m})$ the fifth and the sixth eigenvalues exactly coincide: $\lambda_{0}^{41}=\lambda_{0}^{22}$.

In Reissner-Mindlin equations, this double eigenvalue splits into two different ones, both converging to this common value and hence getting closer and closer to each other as $t$ goes to zero. This phenomenon occurs for both hard and soft simply supported plates. We have chosen the latter (i.e., with vanishing transversal displacements but no constraint imposed on the rotations along the edges) in order to test our method also for these boundary conditions, but similar results are valid for hard simply supported plates too.

We have considered the same values of the physical parameters and successively refined uniform meshes like those of the previous experiment. We have computed approximations $\lambda_{t h}^{41}$ and $\lambda_{t h}^{22}$ on the meshes corresponding to $N=8,16,32,64$, for decreasing values of the thickness $t=0.1,0.01,0.001,0.0001 \mathrm{~m}$. 
TABLE 5. Fifth eigenvalue $\lambda_{t h}^{41}$ (multiplied by $10^{-10}$ ) of ReissnerMindlin equations for a soft simply supported rectangular plate: $2 \mathrm{~m} \times 1 \mathrm{~m}$

\begin{tabular}{|l||c|c|c|c||c||c||c|}
\hline Thickness & $N=8$ & $N=16$ & $N=32$ & $N=64$ & $\alpha$ & $\lambda_{t}^{41}$ & $C_{41}$ \\
\hline$t=0.1$ & 3032.768 & 2801.861 & 2736.523 & 2718.535 & 1.83 & 2711.216 & 2.817 \\
$t=0.01$ & 3670.259 & 3407.412 & 3341.052 & 3322.356 & 1.96 & 3316.899 & 3.180 \\
$t=0.001$ & 3678.556 & 3416.164 & 3351.528 & 3335.415 & 2.02 & 3330.245 & 3.459 \\
$t=0.0001$ & 3678.639 & 3416.253 & 3351.638 & 3335.572 & 2.02 & 3330.405 & 3.464 \\
\hline$t=0$ (extrap.) & 3678.640 & 3416.254 & 3351.640 & 3335.574 & 2.02 & 3330.406 & 3.464 \\
\hline
\end{tabular}

TABLE 6. Sixth eigenvalue $\lambda_{t h}^{22}$ (multiplied by $10^{-10}$ ) of ReissnerMindlin equations for a soft simply supported rectangular plate: $2 \mathrm{~m} \times 1 \mathrm{~m}$

\begin{tabular}{|l||c|c|c|c||c||c||c|}
\hline Thickness & $N=8$ & $N=16$ & $N=32$ & $N=64$ & $\alpha$ & $\lambda_{t}^{22}$ & $C_{22}$ \\
\hline$t=0.1$ & 3039.576 & 2802.288 & 2736.243 & 2718.268 & 1.85 & 2711.174 & 2.994 \\
$t=0.01$ & 3676.006 & 3407.927 & 3341.083 & 3322.346 & 1.98 & 3317.021 & 3.322 \\
$t=0.001$ & 3684.276 & 3416.686 & 3351.582 & 3335.421 & 2.04 & 3330.376 & 3.613 \\
$t=0.0001$ & 3684.359 & 3416.775 & 3351.693 & 3335.579 & 2.04 & 3330.536 & 3.618 \\
\hline$t=0$ (extrap.) & 3684.359 & 3416.776 & 3351.694 & 3335.581 & 2.04 & 3330.537 & 3.618 \\
\hline
\end{tabular}

We have observed that the relative error of the computed eigenvalues roughly behaves again like

$$
\frac{\lambda_{t h}^{m n}-\lambda_{t}^{m n}}{\lambda_{t}^{m n}} \approx C_{m n} h^{\alpha}
$$

with constants $C_{m n}$ depending neither on the thickness $t$ nor on the mesh size $h$ and orders of convergence $\alpha$ close to 2 .

For each thickness $t$ and both vibration modes, we have estimated the orders of convergence $\alpha$, the constants $C_{m n}$ and the exact eigenvalues $\lambda_{t}^{m n}$ by a least square fitting of the computed approximate eigenvalues $\lambda_{t h}^{m n}$, similar to that of the previous experiment. Finally we have also estimated by extrapolation the limit values $\lambda_{0 h}^{m n}=\lim _{t \rightarrow 0} \lambda_{t h}^{m n}$.

We summarize these results for $\lambda_{t h}^{41}$ in Table 5 and for $\lambda_{t h}^{22}$ in Table 6 .

Two conclusions arise immediately from these tables. First, the constants do not deteriorate as the thickness becomes small (and consequently the eigenvalues of Reissner-Mindlin equations get closer). Secondly, the method is locking free and provides very accurate approximations of the eigenvalues of the Kirchhoff equations, in this case, $\lambda_{0}^{41}=\lambda_{0}^{22}=3330.225 \times 10^{10}$.

\section{REFERENCES}

1. B.S. Al Janabi and E. Hinton, A study of the free vibrations of square plates with various edge conditions, in Numerical Methods and Software for Dynamic Analysis of Plates and Shells, E. Hinton, ed., Pineridge Press, Swansea, 1987.

2. D.N. Arnold and R.S. Falk, A uniformly accurate finite element method for the ReissnerMindlin plate, SIAM J. Numer. Anal., 26 (1989) 1276-1290. MR 91c:65068

3. I. Babuška and J. Osborn, Eigenvalue problems, in Handbook of Numerical Analysis, Vol. II, P. G. Ciarlet and J. L. Lions, eds., North Holland, Amsterdam, 1991, pp. 641-787. MR 92f:65001 
4. K.J. Bathe, Finite Element Procedures in Engineering Analysis, Prentice-Hall, Englewood Cliffs, NJ, 1982.

5. K.J. Bathe and F. Brezzi, On the convergence of a four-node plate bending element based on Mindlin/Reissner plate theory and a mixed interpolation, in Mathematics of Finite Elements and Applications V, J.R. Whiteman, ed., Academic Press, London, 1985, pp. 491-503. MR 87f: 65125

6. K.J. Bathe and E.N. Dvorkin, A four-node plate bending element based on Mindlin/Reissner plate theory and a mixed interpolation, Internat. J. Numer. Methods Eng. 21 (1985) 367-383.

7. F. Brezzi and M. Fortin, Mixed and Hybrid Finite Element Methods, Springer-Verlag, New York, 1991. MR 92d:65187

8. F. Brezzi, M. Fortin and R. Stenberg, Quasi-optimal error bounds for approximation of shearstresses in Mindlin-Reissner plate models, Math. Models Methods Appl. Sci. 1 (1991) 125-151. MR 92e: 73030

9. P. Clement, Approximation by finite element functions using local regularization. RAIRO Anal. Numér., 9 (1975) 77-84. MR 53:4569

10. R. Durán and E. Liberman, On mixed finite element methods for the Reissner-Mindlin plate model, Math. Comp., 58 (1992) 561-573. MR 92f:65135

11. D. J. Dawe and O. L Roufaeil, Rayleigh-Ritz vibration analysis of Mindlin plates, J. Sound. Vib., 12 (1980) 345-359.

12. H.C. Huang and E. Hinton, A nine node Lagrangian Mindlin plate element with enhanced shear interpolation, Eng. Comput., 1 (1984) 369-379.

13. T.J.R Hughes, The Finite Element Method: Linear Static and Dinamic Finite Element Analysis, Prentice-Hall, Englewood Cliffs, NJ, 1987. MR 90i:65001

14. T. Kato, Perturbation Theory for Linear Operators, Grandlehren Math. Wiss. 132, Springer Verlag, Berlin, 1966. MR 34:3324

15. P. Peisker and D. Braess, Uniform convergence of mixed interpolated elements for ReissnerMindlin plates, RAIRO Modél. Math. Anal. Numér., 26 (1992) 557-574. MR 93j:73070

16. J. Pitkäranta and M. Suri, Design principles and error analysis for reduced-shear platebending finite elements, Numer. Math., 75 (1996) 223-266. MR 98c:73078

17. P.A. Raviart and J.M. Thomas, A mixed finite element method for second order elliptic problems, in Mathematical Aspects of Finite Element Methods, Lecture Notes in Mathematics 606, Springer Verlag, Berlin, Heidelberg, New York, 1977, pp. 292-315. MR 58:3547

18. L.R. Scott and S. Zhang, Finite element interpolation of nonsmooth functions satisfying boundary conditions, Math. Comp. 54 (1990) 483-493. MR 90j:65021

19. O.C. Zienkiewicz and R.L. Taylor, The Finite Element Method, Vol. 2, McGraw-Hill, 1989.

Departamento de Matemática, Facultad de Ciencias Exactas y Naturales, UniversiDad de Buenos Aires, 1428 Buenos Aires, Argentina

E-mail address: rduran@dm.uba.ar

Departamento de Matemática Aplicada, Universidade de Santiago de Compostela, 15706 Santiago de Compostela, Spain

E-mail address: luisher@zmat.usc.es

Comisión de Investigaciones Científicas de la Provincia de Buenos Aires and Departamento de Matemática, Facultad de Ciencias Exactas, Universidad Nacional de la Plata, C.C. $172 ., 1900$ La Plata, Argentina

E-mail address: elsali@mate.unlp.edu.ar

Departamento de Ingeniería Matemática, Universidad de Concepción, Casilla 4009, CONCEPCión, ChiLe

E-mail address: rodolfo@ing-mat.udec.cl

Departamento de Matemática, Facultad de Ciencias Exactas, Universidad Nacional de La Plata, C.C. 172., 1900 La Plata, Argentina

E-mail address: solo@mate.unlp.edu.ar 